\title{
Effect of phosphorus and bio-fertilizers on productivity and quality of soybean at Siwa Oasis
}

\author{
Abdel - lateef, A.A. \\ Agronomy Unit, Plant Production Dept. Desert Research Center, Mataria, Cairo. Egypt.
}

\begin{abstract}
Tow field experiment were carried out during two successive summer seasons 2013 and 2014 at the Experimental Station at Khamisalocation Siwa Oasis, Desert Research Center(DRC), to study, response of soybean (Glycine max) Giza, 35 cultivar to the combination between phosphorus fertilization, and four levels phosphorus fertilization:(0, 30, 45and60 kg. $\mathrm{P}_{2} \mathrm{O}_{5} / \mathrm{fed}$.). And four spring with four bio-fertilizers treatment (control, bio magic compound at a rate of ( 2 liters / fed.), amino carpi $\mathrm{K}$ compound $\left(200 \mathrm{~cm}^{3} / \mathrm{fed}\right.$.), and Bio greenmeracl compound (1 liters / 200 liters water / fed.).Using the split plot design which, the bio-fertilizers treatment were distributed in the main plots, whereas phosphate fertilization in subplot in four replicates.
\end{abstract}

\section{The obtained results could be summarized as follows:}

The results showed a significant increase in yield and its components, as well as the oil yield / fed. using ground phosphorus fertilization at the rate of $60 \mathrm{~kg} \mathrm{p}_{2} \mathrm{O}_{5} / \mathrm{fed}$. in both seasons respectively.

The three bio- fertilizers of significantly differences were significant and increased the yield and its components as well as the oil crop, and the best bio-fertilizer was a compound Amino Kirby K, $200 \mathrm{~cm}^{3}$, in both seasons. The interaction between phosphorus and bio- fertilizers led to increasing the yield and its component. The best practice at a rate of $60 \mathrm{~kg} \mathrm{p}_{2} \mathrm{O}_{5} / \mathrm{fed}$. + spraying with Amino Kirby K, $200 \mathrm{~cm}^{3} / \mathrm{fed}$., in both seasons. The results showed that phosphorus fertilization at the rate of $60 \mathrm{~kg} \mathrm{p}_{2} \mathrm{O}_{5} / \mathrm{fed}$. and spraying with Amino Kirby $\mathrm{K}$, $200 \mathrm{~cm}^{3} /$ fed.,resulted in significant increase in the chemical components of soybean seeds namely: nitrogen, total protein,oil percentage, phosphorusand potassium, in both seasons.Found from the calculation of the economic yield of crop and oil yield, the use of the high rate of phosphorus fertilization $\left(60 \mathrm{~kg}\right.$. $\mathrm{P}_{2} \mathrm{O}_{5} / \mathrm{fed}$.) and spraying with amino carpi $\mathrm{K}$ compound $\left(200 \mathrm{~cm}^{3} /\right.$ fed.) is the best experimental economic transaction for the farmer under the conditions of Siwa Oasis, especially that the crop of soybeans is grown for the first time in the oasis.

Key words: soybean, phosphorus, bio-fertilizers, Siwa Oasis

\section{Introduction}

Siwa oasis in the western desert of Egypt between longitude $25^{\circ} 18^{\prime}$ and $26^{\circ} 06^{\prime} \mathrm{E}$ and latitudes $29^{\circ} 05^{\prime}$ and $29^{\circ} 20^{\prime} \mathrm{N}$, is the most well known of the five major oasis in the Western desert of Egypt and is one of the most promising areas for adding new agricultural land. Most of the new reclaimed areas in Egypt are sandy and loamy sand soil suitable for planting with many crops. The use of bio-fertilizers that have an impact on the increase rate of flowering, as well as the contract, and also the fullness of pods and grains, The combined effect of phosphorus and biological fertilization on the productivity and quality of soybeans.

Soybean seeds are a major source of protein $(40 \%)$ and oil (20\%) for human consumptions. Soybean oil is one of the common vegetable oils containing a significant amount of unsaturated fatty acids: linolenic acid; linoleic acid (omega-6 acid) and oleic acid (, (Yaklich et al. 2002). Also, soybean is one of the most important summer leguminous crops, extensively successful in many provinces in Egypt; Soybean seed production may be limited by environmental stresses such as soil salinity (Ghassemi-Golezani et al., 2009).Lehmann et al. (2010) reported that the proline is an important multifunctional amino acid and plays a role in carbon and nitrogen metabolism, cell signaling, nutrient adaptation and protection against osmotic and oxidative stresses. Sayari et al (2005) found that the proline accumulation in response to drought or salinity stress has been reported to occur in the cytosol to adjust the osmotic balance. Sessitch $\boldsymbol{e t}$ al. (2002) found that Rhizobium ssp. i.e. rhizobactria and some are endophytes which can produce phytohormones, siderophores, solublitizespringly soluble organic and inorganic phosphates and can colnize the roots. Concerning that the importance of soybean in production of oil, its nutritional important and status of biological fertilizers in sustainable agriculture.

The use of fertilizer is considered to be one of the most important factors in increasing crop yield. Phosphorous has been shown to be an essential element and its application has been shown to be important for growth, development and yield of soybean (Kakar et al., 2002). Also, phosphorus is an important plant nutrient involved in several energy transformation and biochemical reactions including nitrogen fixation. Phosphorus deficiency can cause a limit nodulation in legumes and $\mathrm{P}$ fertilizer application can also overcome this deficiency (Carsky et al., 2001). The use of phosphate 
solubilizing bacteria is able to change insoluble phosphorus in soil into the absorbed soluble form (Turanet al., 2006). El-Gizawy and Mehasen (2009) showed that application of mineral phosphorus fertilizer with phosphate- solubilizing bacteria had a significant effect on bean seed yield and its components, nitrogen, phosphorus and zinc content in seeds. Seeds inoculated with bio fertilizers in combination with foliar application of micronutrients gave higher values of all estimated parameters of pea bean than plants fertilized with chemical fertilizers in the presence or absence of foliar application (Zaghloulet al., 2015). In all cases, fertilizers are major factors in maintaining soil fertility, but using too much of them, greatly reduce the amount of soil microorganisms. Environmental problems caused by irregular application of chemical fertilizers have harmful effects on biological cycles and destroyed farming stability systems; these factors altogether encourage the application of bio fertilizers (Kannayan, 2002). Integration of chemical and bio fertilizers is one of the ways to increase production in sustainable agriculture (Ali et al., 2008). From otherwise, one biological way to increase the productivity in the agricultural sector is the effective use of beneficial microorganisms that have more ability to enhance plant growth and yield.

Therefore the present investigation was aimed to study the productivity of Soybean (Giza, 35 cultivar) to combination between Phosphate fertilizer, and four types of bio-fertilizers on yield and its components under Siwa Oasis conditions.

\section{Materials and Methods}

Tow field experiment were carried out during two successive summer seasons 2013 and 2014 at the Experimental station ofat Khamisalocation Siwa Oasis, Desert Research Center, to study, the response of Soybean (Giza, 35 cultivar) to the combination between four phosphorus fertilization, 0,30,45 and 60 kg. $\mathrm{P}_{2} \mathrm{O}_{5} /$ fed. as a soil applicationas affected by four in spraying of types of bio-fertilizers is as follows: (spraying with tap water, bio magic compound 2 liters / fed., amino carpi $\mathrm{K}$ compound $200 \mathrm{~cm}^{3} /$ fed.and Bio greenmeracl compound 1 liter / fed.

A split plot design with four replicates was used. The main plots were devoted to the bio-fertilizers treatments and sub plots were devoted to the Phosphorous fertilizer levels.Each experimental unit contained 5 in ridges $2.5 \mathrm{~m}$ long and $60 \mathrm{~cm}$ apart. The seeds were sowing on April $10^{\text {th }}$ both seasons. Before sowing all plots received $30 \mathrm{~m}^{3} /$ fed. Organic fertilizer. In addition, $100 \mathrm{~kg}$ ammonium sulphate $(20.5 \% \mathrm{~N})$ and $200 \mathrm{~kg}$ potassium sulphate / fed. $\left(48 \% \mathrm{~K}_{2} \mathrm{O}\right)$ were applied at two doses after two and three weeks from sowing, Agriculture was carried out on both sides of the line and the distance between the dots was $30 \mathrm{~cm}$, fertilization was added in three batches between the batch and the other 20 days after planting, respectively.

Mechanical and chemical analysis of the experimental soil is shown in Tables (1).

The soil analysis was carried out according to

Jackson (1967).

Table 1. Mechanical properties of the experimental soil at Khamisa research station.

\begin{tabular}{|c|c|c|c|c|c|c|c|c|c|c|c|}
\hline \multirow{2}{*}{\multicolumn{2}{|c|}{ Depth $(\mathrm{cm})$}} & \multicolumn{7}{|c|}{ Particle size distribution (\%) } & \multirow{2}{*}{\multicolumn{3}{|c|}{ Soil texture }} \\
\hline & & \multicolumn{2}{|r|}{ Coarse sand } & \multicolumn{2}{|r|}{ Fine sand } & \multicolumn{2}{|r|}{ Silt } & Clay & & & \\
\hline \multirow{2}{*}{\multicolumn{2}{|c|}{$\begin{array}{c}0-30 \\
30-60 \\
\end{array}$}} & \multirow{2}{*}{\multicolumn{2}{|c|}{$\begin{array}{l}46.8 \\
50.0 \\
\end{array}$}} & & 28.2 & & 15.4 & \multirow{2}{*}{\multicolumn{2}{|c|}{$\begin{array}{l}9.6 \\
6.1\end{array}$}} & \multicolumn{2}{|c|}{ Sandy loam } \\
\hline & & & & & 25.9 & & 18.0 & & & \multicolumn{2}{|c|}{ Sandy loam } \\
\hline \multicolumn{12}{|c|}{ Chemical analyses. } \\
\hline \multirow{3}{*}{$\begin{array}{l}\text { Depth } \\
(\mathrm{cm})\end{array}$} & \multirow{3}{*}{$\mathrm{pH}$} & \multirow{3}{*}{$\begin{array}{c}\text { Ec } \\
(\mathrm{dS} / \mathrm{m})\end{array}$} & \multirow{3}{*}{$\begin{array}{c}\mathrm{O} . \mathrm{M} \\
\%\end{array}$} & \multicolumn{8}{|c|}{ Saturation soluble extract } \\
\hline & & & & \multicolumn{4}{|c|}{ Soluble anions (meq / L) } & \multicolumn{4}{|c|}{ Soluble cations (meq / L) } \\
\hline & & & & $\mathrm{Co}_{3}^{-}$ & $\mathrm{HCO}_{3}^{-}$ & $\mathrm{SO}_{4}^{-}$ & $\mathrm{Cl}^{-}$ & $\mathrm{Ca}^{++}$ & $\mathrm{Mg}^{++}$ & $\mathrm{Na}^{+}$ & $\mathrm{K}^{+}$ \\
\hline $0-30$ & 7.4 & 12.32 & 0.7 & - & 2.8 & 26.8 & 70.4 & 30.7 & 17.24 & 49.6 & 1.31 \\
\hline $30-60$ & 7.8 & 13.04 & 0.5 & - & 3.0 & 20.5 & 76.5 & 26.2 & 15.8 & 57.3 & 0.7 \\
\hline
\end{tabular}

Regular irrigation was carried out in the whole experiment every one week after sowing. The meteorological data of Khamisa location was shows in Table (2).

Soybean growth and yield of the two inner ridges were determined for each sub plot and a sample of five plants were taken at harvesting lime at random to estimate the following characters: plant height $(\mathrm{cm}$.$) , No. of pods /plant, pod length$ (cm.),pods weight / plant (g.), No. of seeds / plant, seed weight / plant (g.), 100 - seed weight (g.),seed,straw and biological yields and (chemical composition of seeds i.e., nitrogen, phosphorus, potassium, total crud protein and oil content which estimated by using according to A.O.A.C. (1975). Oil and protein yields (Kg/fed) was determined by multiplying seed yield (Kg/fed) by seed oil percentage nitrogen $\%$ × $6.25=$ protein $\%$, protein $\%$ x seed yield=protein yield/ fed.and oil yield. All the obtained data were subjected to statistical analysis, the two seasons. The mean values were compared according to the procedures of analysis of variance (ANOVA) by using LSD at the level of 5\% of significance according to Snedecor and Cochran (1980). 
Table 2. Meteorological data of temperature $\left({ }^{\circ} \mathrm{C}\right)$, relative humidity $(\%)$, and Relative Humidity $(\%)$ of Khamisalocation

\begin{tabular}{|c|c|c|c|c|c|c|}
\hline \multirow{2}{*}{ Months } & \multicolumn{2}{|c|}{$\begin{array}{l}\text { Average temperature. } \\
\qquad\left({ }^{\circ} \mathrm{C}\right)\end{array}$} & \multicolumn{2}{|c|}{ Average rain full (mm) } & \multicolumn{2}{|c|}{$\begin{array}{l}\text { Average relative humidity } \\
(\%)\end{array}$} \\
\hline & 2013 & 2014 & 2013 & 2014 & 2013 & 2014 \\
\hline March & 27.35 & 28.14 & - & - & 75.66 & 77.33 \\
\hline April & 34.84 & 34.51 & - & - & 77.25 & 78.18 \\
\hline May & 37.78 & 38.25 & - & - & 81.42 & 83.44 \\
\hline Jun & 39.57 & 41.40 & - & - & 83.29 & 84.06 \\
\hline July & 41.32 & 43.55 & - & - & 85.41 & 87.63 \\
\hline August & 41.45 & 42.78 & - & - & 84.62 & 86.08 \\
\hline September & 38.12 & 39.22 & - & - & 82.11 & 83.60 \\
\hline
\end{tabular}

\section{Economic Assessment:}

A comprehensive economic assessment of the experiment (for both inputs and outputs of the experiment) is performed.

\section{Results and Discussion}

\section{I: - Yield and yield components.}

The results obtained from tables (3and 4) showed that soil additions of phosphorus fertilization resulted in a significant increase in the yield. From to the height yield was obtained from rate of $60 \mathrm{~kg} \mathrm{p}_{2} \mathrm{O}_{5} /$ fed. compared with the rest of the other treatments in yield and its components, plant height, number of pods /plant, pod length, pods weight / plant, number of seeds / plant, seed weight / plant, 100 - seed weight,seed,straw as well as biological yields, and oil and protein yields. These results are agreement with those obtained by Kakar, et. al.(2002) and ElGizawy and Mehasen (2009). Also, the results also showed that the use of spraying with different types of bio-fertilizers increasing the yield and its components combination withspraying by tap water. The best values in yield characteristics and its components were due to the addition of the aminokarbi K compound (200 cm / fed.), Then the lower of the Bio greenmeracl compound (1 liters / fed.). anda biomagic compound at a rate of (2 liters / fed.), While the lowest values were for the un- biological fertilizer treatment. It may be noted that the soybean crop of leguminous crops that require phosphorus and the amino-karbi $\mathrm{K}$ compound contains this element, which increases the flowering rate - as well as the contract and thus works to fill the pods and seeds.

The interaction between soil applications for phosphorus fertilizer at a rate of $60 \mathrm{~kg} \mathrm{P}_{2} \mathrm{O}_{5} /$ fed. and spraying by Amino karbi K. compound at a rate of (200 $\mathrm{cm}^{3} / 200$ liters of water/fad.) gave the highest values in yield and its components, while the lowest values were when using the treatment without adding ground for phosphorus fertilization with spraying and tap water. The percentage of increase in these characteristics was: $18.34 \%, 19.21 \%, 18.19 \%$, $20.10 \%, 21.64 \%, 20.44 \%, 21.17 \%, 22.11 \%, 18.96 \%$, $17.34 \%$ and $18.81 \%$, respectively. In this respect,
Amin and Ezatollah (2014). Found that the combined effect of phosphorus and bio-fertilization on the productivity and quality of soybeans increased the proportion and quality of soybean oil using. Contract, and also the fullness of centuries and grains, mineral fertilization (phosphate) balanced increases the proportion of oil.These results were obtained by Yaklich, et. al. (2002), Abdelhamid and El-Metwally, (2008), Abdel-Fattah, et.al. (2011), Iraj, et.al.(2012) and Amin and Ezatollah (2014).

\section{II: -chemical composition of soybean seeds:}

Results in Table (5) Showed that the chemical analysis of soybean seeds showed phosphorus fertilizers a significant increase in the study characteristics, i.e., nitrogen, phosphorus, potassium, total crud protein and oil percentages compared with un fertilizer treatment. With the addition of the rate of $60 \mathrm{~kg} . \mathrm{P}_{2} \mathrm{O}_{5} /$ fed. led to a significant increase in the proportion of oil in the seeds, which had a clear effect in the oil yield, as well as the increase in the percentage of total protein by a clear percentage of the rest of the transactions. The results showed that the use of spraying by Amino karbi K. compound at a rate of $\left(200 \mathrm{~cm}^{3} /\right.$ fed. $)$. Significant differences the rests of the other biochemical compounds in all studied traits. Similar results were obtained by Agwu, et. al. (2009), El-Gizawy and Mehasen (2009), Gordana, et. al.(2014) and Amin and Ezatollah (2014).

The interaction between soil applications for phosphorus fertilizers at a rate of $60 \mathrm{~kg}_{2} \mathrm{O}_{5} / \mathrm{fed}$. and spraying by amino karbi $\mathrm{K}$. compound at a rate of $200 \mathrm{~cm}^{3} /$ fed.gave the highest values of the chemical analysis of soybean seeds. The percentage of increase in the qualities under study characteristics, i.e., nitrogen, phosphorus, potassium, total crud protein and oil. $11.34 \%, 12.58 \%, 10.95 \%$, $14.59 \%$ and $14.66 \%$, respectively as compared significantly increased all chemical composition tharl the other biofertilizer. Similar results were obtained by Sayari, et. al. (2005), Abdelhamid and ElMetwally, (2008), Ali, et. al. (2008), and Manal, et. al. (2014). 
Table 3. Effect of phosphorus and bio-fertilizers application of soybean on yield and its components of 2013 and 2014 growing seasons.

\begin{tabular}{|c|c|c|c|c|c|c|c|c|c|c|c|}
\hline \multirow{2}{*}{\multicolumn{2}{|c|}{$\begin{array}{l}\text { Characters } \\
\text { treatment }\end{array}$}} & \multicolumn{2}{|c|}{ Plant height(cm.) } & \multicolumn{2}{|c|}{$\begin{array}{l}\text { Number of } \\
\text { pods /plant }\end{array}$} & \multicolumn{2}{|c|}{$\begin{array}{l}\text { Pod length } \\
\text { (cm.) }\end{array}$} & \multicolumn{2}{|c|}{$\begin{array}{l}\text { Number of seeds } \\
\text { / plant }\end{array}$} & \multicolumn{2}{|c|}{$\begin{array}{l}\text { Pods weight / } \\
\text { plant (g.) }\end{array}$} \\
\hline & & $1^{\text {st. }}$. & $\overline{2^{\text {nd }}}$ & $1^{\text {st. }}$ & $2^{\text {nd }}$ & $1^{\text {st. }}$ & $2^{\text {nd }}$ & 1st. $^{\text {st. }}$ & $2^{\text {nd }}$ & $\mathbf{1}^{\text {st }}$. & $2^{\text {nd }}$. \\
\hline \multirow{4}{*}{ Bio.1 } & P1 & 84.12 & 83.78 & 43.01 & 42.18 & 5.00 & 5.01 & 98.54 & 99.54 & 18.22 & 19.67 \\
\hline & $\mathbf{P 2}$ & 86.54 & 85.67 & 44.55 & 45.09 & 5.09 & 5.10 & 102.14 & 104.11 & 19.04 & 20.22 \\
\hline & P3 & 88.95 & 89.15 & 45.00 & 46.22 & 5.67 & 5.77 & 108.56 & 107.55 & 20.55 & 21.00 \\
\hline & P4 & 91.24 & 91.58 & 46.28 & 46.22 & 5.69 & 5.88 & 111.50 & 112.50 & 21.55 & 22.02 \\
\hline Mean & & 87.71 & 87.54 & 44.71 & 44.92 & 5.36 & 5.44 & 105.18 & 105.93 & 19.84 & 20.73 \\
\hline \multirow{4}{*}{ Bio.2 } & P1 & 89.57 & 88.97 & 45.88 & 45.67 & 5.66 & 5.72 & 107.68 & 108.64 & 19.98 & 20.88 \\
\hline & $\mathbf{P 2}$ & 98.32 & 99.12 & 48.66 & 48.76 & 6.11 & 6.21 & 114.80 & 114.00 & 21.20 & 22.98 \\
\hline & P3 & 104.66 & 105.32 & 52.22 & 55.66 & 6.25 & 6.22 & 122.47 & 123.12 & 23.32 & 24.65 \\
\hline & P4 & 111.55 & 115.84 & 59.58 & 58.64 & 6.76 & 6.22 & 134.21 & 135.64 & 25.94 & 26.76 \\
\hline Mean & & 101.03 & 102.31 & 51.59 & 52.18 & 6.19 & 6.09 & 119.79 & 120.35 & 22.61 & 23.82 \\
\hline \multirow{4}{*}{ Bio.3 } & P1 & 102.55 & 101.31 & 58.14 & 57.25 & 6.48 & 6.55 & 124.81 & 123.25 & 23.32 & 24.54 \\
\hline & $\mathbf{P 2}$ & 109.54 & 110.55 & 60.21 & 61.00 & 6.89 & 6.99 & 134.66 & 135.87 & 25.58 & 26.57 \\
\hline & P3 & 111.88 & 114.00 & 63.00 & 64.85 & 7.54 & 7.66 & 142.54 & 143.55 & 27.48 & 28.34 \\
\hline & P4 & 115.22 & 121.02 & 66.57 & 65.47 & 8.01 & 8.21 & 155.21 & 154.87 & 31.57 & 30.86 \\
\hline Mean & & 109.79 & 111.72 & 61.98 & 62.14 & 7.23 & 7.35 & 139.31 & 139.39 & 26.99 & 27.57 \\
\hline \multirow{4}{*}{ Bio.4 } & $\mathbf{P 1}$ & 103.54 & 102.99 & 52.01 & 53.01 & 5.98 & 6.01 & 108.22 & 109.11 & 20.18 & 21.07 \\
\hline & $\mathbf{P 2}$ & 105.64 & 105.66 & 53.87 & 54.45 & 6.87 & 6.81 & 115.61 & 115.00 & 22.56 & 23.57 \\
\hline & P3 & 108.94 & 108.00 & 56.04 & 55.21 & 6.77 & 6.89 & 124.00 & 124.95 & 25.88 & 25.88 \\
\hline & P4 & 109.54 & 108.44 & 60.18 & 57.64 & 7.00 & 6.84 & 132.58 & 133.87 & 27.00 & 27.61 \\
\hline Mean & & 106.91 & 106.27 & 55.52 & 55.08 & 6.65 & 6.63 & 120.10 & 120.73 & 23.91 & 24.53 \\
\hline \multirow{4}{*}{$\mathrm{P}_{2} \mathrm{O}_{5}$} & P1 & 94.94 & 94.26 & 49.76 & 49.52 & 5.78 & 5.82 & 109.81 & 110.13 & 20.42 & 21.54 \\
\hline & $\mathbf{P 2}$ & 100.01 & 100.25 & 51.82 & 52.32 & 6.24 & 6.27 & 116.80 & 117.24 & 22.09 & 23.33 \\
\hline & P3 & 103.60 & 104.11 & 54.06 & 55.48 & 6.55 & 6.63 & 124.39 & 124.79 & 24.20 & 24.96 \\
\hline & P4 & 106.88 & 109.22 & 58.15 & 56.99 & 6.86 & 6.78 & 133.37 & 134.22 & 26.51 & 26.81 \\
\hline \multicolumn{2}{|l|}{ Mean } & 101.35 & 101.96 & 53.44 & $\mathbf{5 3 . 5 7}$ & 6.25 & 6.37 & 121.09 & 121.59 & 23.30 & 24.16 \\
\hline \multirow{3}{*}{\multicolumn{2}{|c|}{$\begin{array}{c}\text { LSD } 5 \% \text { P. } \\
\text { Bio. } \\
\text { PxBio }\end{array}$}} & 2.012 & 2.028 & 0.658 & 0.661 & 0.023 & 0.026 & 2.012 & 2.019 & 1.002 & 1.014 \\
\hline & & 2.022 & 2.023 & 0.651 & 0.658 & 0.022 & 0.023 & 2.008 & 2.011 & 1.001 & 1.010 \\
\hline & & 1.892 & 1.976 & 0.422 & 0.549 & 0.018 & 0.020 & 1.867 & 1.908 & 0.982 & 0.984 \\
\hline
\end{tabular}


Table 4. Effect of phosphorus and bio-fertilizers application of soybean on yield and its components and oil yield of 2013 and 2014 growing seasons.

\begin{tabular}{|c|c|c|c|c|c|c|c|c|c|c|c|c|c|}
\hline \multirow{2}{*}{\multicolumn{2}{|c|}{$\begin{array}{l}\text { Characters } \\
\text { treatment }\end{array}$}} & \multicolumn{2}{|c|}{$\begin{array}{l}\text { Seed weight } \\
\text { / plant (g.) }\end{array}$} & \multicolumn{2}{|c|}{$\begin{array}{l}100 \text { - Seed } \\
\text { weight (g.) }\end{array}$} & \multicolumn{2}{|c|}{$\begin{array}{l}\text { Biological } \\
\text { yield (Ton } \\
\text { /Fed.) }\end{array}$} & \multicolumn{2}{|c|}{$\begin{array}{l}\text { Seed yield } \\
\text { (Ton /Fed.) }\end{array}$} & \multicolumn{2}{|c|}{$\begin{array}{l}\text { Straw yield } \\
\text { (Ton /Fed.) }\end{array}$} & \multicolumn{2}{|c|}{$\begin{array}{c}\text { Oil yield } \\
\text { (Ton /Fed.) }\end{array}$} \\
\hline & & $1^{\text {st }}$ & $2^{\text {nd. }}$. & $1^{\text {st }}$ & $2^{\text {nd }}$. & $1^{\text {st. }}$ & $2^{\text {nd }}$ & $1^{\text {st. }}$ & $2^{\text {nd }}$ & $1^{\text {st. }}$ & $2^{\text {nd }}$. & $1^{\text {st. }}$. & $2^{\text {nd }}$ \\
\hline \multirow{4}{*}{ Bio.1 } & P1 & 16.66 & 17.20 & 14.65 & 14.25 & 2.263 & 2.135 & 0.882 & 0.834 & 1.381 & 1.301 & 0.175 & 0.166 \\
\hline & P2 & 18.24 & 18.96 & 15.07 & 15.10 & 2.439 & 2.449 & 0.938 & 0.942 & 1.501 & 1.507 & 0.197 & 0.199 \\
\hline & P3 & 19.57 & 19.67 & 15.15 & 15.24 & 2.545 & 2.592 & 0.994 & 0.997 & 1.551 & 1.595 & 0.212 & 0.213 \\
\hline & P4 & 19.99 & 20.21 & 15.34 & 15.33 & 2.575 & 2.751 & 1.006 & 1.058 & 1.569 & 1.693 & 0.227 & 0.239 \\
\hline Mean & & 18.61 & 19.01 & 15.05 & 14.98 & 2.455 & 2.481 & 0.955 & 0.957 & 1.500 & 1.524 & 0.202 & 0.204 \\
\hline \multirow{4}{*}{ Bio.2 } & P1 & 18.25 & 19.85 & 14.88 & 15.00 & 2.442 & 2.538 & 0.954 & 0.976 & 1.488 & 1.561 & 0.190 & 0.196 \\
\hline & P2 & 20.65 & 21.55 & 15.22 & 15.34 & 2.553 & 2.623 & 0.997 & 1.009 & 1.555 & 1.614 & 0.222 & 0.225 \\
\hline & P3 & 21.86 & 23.09 & 15.65 & 15.69 & 2.821 & 2.891 & 1.102 & 1.112 & 1.719 & 1.779 & 0.262 & 0.266 \\
\hline & P4 & 23.54 & 24.00 & 16.22 & 16.34 & 3.215 & 3.260 & 1.256 & 1.254 & 1.959 & 2.006 & 0.300 & 0.300 \\
\hline Mean & & 21.07 & 22.12 & 15.49 & 15.59 & 2.757 & 2.828 & 0.829 & 1.088 & 1.680 & 1.740 & 0.243 & 0.246 \\
\hline \multirow{4}{*}{ Bio.3 } & P1 & 22.11 & 22.89 & 15.34 & 15.66 & 2.524 & 2.602 & 0.986 & 1.001 & 1.538 & 1.601 & 0.200 & 0.214 \\
\hline & P2 & 24.16 & 24.88 & 16.24 & 16.34 & 3.066 & 3.091 & 1.198 & 1.189 & 1.868 & 1.902 & 0.259 & 0.269 \\
\hline & P3 & 26.55 & 26.95 & 17.44 & 17.60 & 3.297 & 3.409 & 1.288 & 1.311 & 2.009 & 2.097 & 0.289 & 0.315 \\
\hline & P4 & 28.57 & 28.99 & 18.00 & 17.85 & 3.724 & 3.762 & 1.455 & 1.447 & 2.269 & 2.315 & 0.372 & 0.363 \\
\hline Meal & & 25.34 & 25.93 & 16.75 & 16.86 & 3.152 & 3.216 & 1.231 & 1.237 & 1.921 & 1.978 & 0.281 & 0.292 \\
\hline \multirow{4}{*}{ Bio.4 } & P1 & 20.14 & 20.97 & 14.65 & 14.56 & 2.427 & 2.566 & 0.948 & 0.987 & 1.479 & 1.579 & 0.190 & 0.197 \\
\hline & P2 & 22.65 & 21.54 & 15.07 & 15.10 & 2.563 & 2.673 & 1.001 & 1.028 & 1.562 & 1.649 & 0.212 & 0.219 \\
\hline & P3 & 23.97 & 23.00 & 15.55 & 15.73 & 3.039 & 3.115 & 1.187 & 1.198 & 1.852 & 1.917 & 0.266 & 0.271 \\
\hline & P4 & 25.22 & 24.58 & 16.55 & 16.87 & 3.196 & 3.377 & 1.248 & 1.299 & 1.947 & 2.078 & 0.298 & 0.305 \\
\hline prean & & 22.99 & 22.52 & 15.43 & 15.57 & 2.806 & 2.932 & 1.096 & 1.128 & 1.710 & 1.805 & 0.241 & 0.248 \\
\hline \multirow{4}{*}{$\mathbf{P}_{2} \mathrm{O}_{5}$} & P1 & 19.29 & 20.22 & 14.88 & 14.86 & 2.414 & 2.460 & 0.942 & 0.949 & 1.471 & 1.510 & 0.188 & 0.193 \\
\hline & P2 & 21.42 & 21.73 & 15.40 & 15.47 & 2.655 & 2.705 & 1.033 & 1.042 & 1.621 & 1.668 & 0.222 & 0.228 \\
\hline & P3 & 22.98 & 23.17 & 15.94 & 16.06 & 2.922 & 3.001 & 1.142 & 1.154 & 1.782 & 1.847 & 0.257 & 0.266 \\
\hline & P4 & 24.32 & 24.44 & 16.52 & 16.59 & 3.177 & 3.287 & 1.241 & 1.264 & 1.936 & 2.022 & 0.299 & 0.293 \\
\hline Mean & & 22.00 & 22.39 & 15.68 & 15.74 & 2.792 & 2.854 & 1.089 & 1.102 & 1.702 & 1.762 & 0.241 & 0.245 \\
\hline \multirow{3}{*}{\multicolumn{2}{|c|}{$\begin{array}{c}\text { LSD 5\% P. } \\
\text { Bio. } \\
\text { PxBio }\end{array}$}} & 1.029 & 1.032 & 0.068 & 0.072 & 0.077 & 0.078 & 0.056 & 0.057 & 0.062 & 0.064 & 0.035 & 0.038 \\
\hline & & 1.022 & 1.028 & 0.067 & 0.069 & 0.074 & 0.076 & 0.052 & 0.054 & 0.060 & 0.061 & 0.033 & 0.035 \\
\hline & & 0.998 & 1.003 & 0.059 & 0.062 & 0.064 & 0.066 & 0.048 & 0.048 & 0.049 & 0.052 & 0.021 & 0.024 \\
\hline
\end{tabular}


Table 5. Effect of phosphorus and bio-fertilizers application of soybean on chemical composition of the seeds in 2013 and 2014 growing seasons.

\begin{tabular}{|c|c|c|c|c|c|c|c|c|c|c|c|c|c|}
\hline \multirow{2}{*}{\multicolumn{2}{|c|}{$\begin{array}{l}\text { Characters } \\
\text { treatment }\end{array}$}} & \multicolumn{2}{|c|}{$\begin{array}{c}\text { Protein yield } \\
\text { (Ton /Fed.) }\end{array}$} & \multicolumn{2}{|c|}{$\begin{array}{c}\text { Nitrogen } \\
(\%)\end{array}$} & \multicolumn{2}{|c|}{$\begin{array}{c}\text { Phosphorus } \\
(\%)\end{array}$} & \multicolumn{2}{|c|}{$\begin{array}{c}\text { Potassium } \\
(\%)\end{array}$} & \multicolumn{2}{|c|}{$\begin{array}{c}\text { Total crud } \\
\text { protein }(\%)\end{array}$} & \multicolumn{2}{|c|}{ Oil content (\%) } \\
\hline & & $\mathbf{1}^{\mathrm{st}}$ & $2^{\text {nd }}$ & $\mathbf{1}^{\mathrm{st}}$ & $2^{\text {nd }}$ & $\mathbf{1}^{\mathrm{st}}$ & $2^{\text {nd }}$ & $\mathbf{1}^{\mathrm{st}}$. & $2^{\text {nd }}$. & $\mathbf{1}^{\mathrm{st}}$ & $2^{\text {nd }}$ & $\mathbf{1}^{\mathrm{st}}$. & $2^{\text {nd }}$. \\
\hline & P1 & 0.281 & 0.266 & 5.11 & 5.07 & 0.310 & 0.314 & 2.122 & 2.125 & 29.53 & 29.25 & 19.92 & 19.94 \\
\hline \multirow[t]{3}{*}{ Bio.1 } & P2 & 0.305 & 0.306 & 5.21 & 5.20 & 0.316 & 0.317 & 2.149 & 2.155 & 30.22 & 30.16 & 21.02 & 21.04 \\
\hline & P3 & 0.332 & 0.333 & 5.36 & 5.39 & 0.321 & 0.324 & 2.178 & 2.198 & 31.09 & 31.26 & 21.34 & 21.35 \\
\hline & P4 & 0.348 & 0.370 & 5.55 & 5.60 & 0.345 & 0.344 & 2.180 & 2.200 & 32.19 & 32.48 & 22.58 & 22.55 \\
\hline \multirow[t]{3}{*}{ Mean } & & 0.316 & 0.318 & 5.31 & 5.33 & 0.325 & 0.324 & 2.157 & 2.170 & 30.76 & 30.80 & 21.21 & 21.24 \\
\hline & P1 & 0.314 & 0.323 & 5.28 & 5.30 & 0.319 & 0.320 & 2.167 & 2.187 & 30.62 & 30.74 & 20.01 & 20.05 \\
\hline & P2 & 0.334 & 0.341 & 5.39 & 5.41 & 0.328 & 0.335 & 2.238 & 2.298 & 31.26 & 31.38 & 22.29 & 22.32 \\
\hline \multirow[t]{2}{*}{ Bio. 2} & P3 & 0.391 & 0.394 & 5.68 & 5.67 & 0.357 & 0.368 & 2.318 & 2.355 & 32.94 & 32.89 & 23.79 & 23.88 \\
\hline & P4 & 0.469 & 0.460 & 5.98 & 5.88 & 0.367 & 0.372 & 2.524 & 2.533 & 34.68 & 34.10 & 23.89 & 23.95 \\
\hline \multirow[t]{3}{*}{ Mean } & & 0.379 & 0.379 & 5.58 & 5.55 & 0.342 & 0.348 & 2.311 & 2.343 & 32.37 & 32.27 & 22.49 & 22.56 \\
\hline & P1 & 0.336 & 0.345 & 5.46 & 5.52 & 0.329 & 0.334 & 2.211 & 2.311 & 31.67 & 32.01 & 20.33 & 21.40 \\
\hline & P2 & 0.431 & 0.433 & 5.76 & 5.84 & 0.342 & 0.350 & 2.310 & 2.398 & 33.41 & 33.87 & 21.58 & 22.66 \\
\hline \multirow[t]{2}{*}{ Bio.3 } & P3 & 0.490 & 0.508 & 6.09 & 6.21 & 0.359 & 0.379 & 2.555 & 2.589 & 35.32 & 36.02 & 22.46 & 23.99 \\
\hline & P4 & 0.622 & 0.628 & 6.84 & 6.95 & 0.389 & 0.398 & 2.787 & 2.700 & 39.67 & 40.31 & 25.56 & 25.09 \\
\hline \multirow[t]{3}{*}{ Mean } & & 0.469 & 0.478 & 6.03 & 6.13 & 0.354 & 0.365 & 2.465 & 2.500 & 35.01 & 35.55 & 22.48 & 23.29 \\
\hline & P1 & 0.314 & 0.330 & 5.30 & 5.36 & 0.318 & 0.321 & 2.198 & 2.211 & 30.74 & 31.09 & 20.00 & 20.91 \\
\hline & $\mathbf{P 2}$ & 0.341 & 0.357 & 5.46 & 5.57 & 0.328 & 0.339 & 2.268 & 2.298 & 31.67 & 32.31 & 21.22 & 21.83 \\
\hline \multirow[t]{2}{*}{ Bio.4 } & P3 & 0.408 & 0.432 & 5.50 & 5.78 & 0.355 & 0.359 & 2.455 & 2.387 & 31.90 & 33.52 & 22.42 & 22.76 \\
\hline & P4 & 0.510 & 0.514 & 6.55 & 6.34 & 0.365 & 0.376 & 2.554 & 2.511 & 37.99 & 36.77 & 23.88 & 23.85 \\
\hline \multirow[t]{3}{*}{ Mean } & & 0.393 & 0.408 & 5.70 & 5.77 & 0.341 & 0.349 & 2.368 & 2.351 & 33.07 & 33.42 & 21.88 & 22.33 \\
\hline & P1 & 0.310 & 0.314 & 5.28 & 5.31 & 0.319 & 0.322 & 2.174 & 2.208 & 30.64 & 30.77 & 20.06 & 20.57 \\
\hline & P2 & 0.351 & 0.358 & 5.45 & 5.50 & 0.328 & 0.335 & 2.241 & 2.287 & 31.64 & 31.93 & 21.52 & 21.96 \\
\hline \multirow[t]{2}{*}{$\mathbf{P}_{2} \mathrm{O}_{5}$} & P3 & 0.403 & 0.415 & 5.65 & 5.76 & 0.348 & 0.357 & 2.376 & 2.382 & 32.81 & 33.42 & 22.50 & 22.99 \\
\hline & P4 & 0.483 & 0.489 & 6.23 & 6.19 & 0.366 & 0.372 & 2.511 & 2.486 & 36.13 & 35.10 & 23.97 & 23.86 \\
\hline \multicolumn{2}{|l|}{ Mean } & 0.386 & 0.493 & 5.65 & 5.69 & 0.340 & 0.346 & 2.325 & 2.340 & 32.805 & 32.805 & 22.014 & 22.595 \\
\hline \multicolumn{2}{|c|}{ LSD $5 \%$ P. } & 0.089 & 0.086 & 0.123 & 0.121 & 0.0079 & 0.0084 & 0.049 & 0.048 & 0.864 & 0.873 & 0.987 & 0.982 \\
\hline \multicolumn{2}{|l|}{ Bio. } & 0.082 & 0.079 & 0.119 & 0.114 & 0.0073 & 0.0081 & 0.045 & 0.044 & 0.852 & 0.859 & 0.973 & 0.964 \\
\hline \multicolumn{2}{|c|}{ PxBio } & 0.073 & 0.071 & 0.102 & 0.101 & 0.0066 & 0.0071 & 0.035 & 0.036 & 0.658 & 0.684 & 0.867 & 0.786 \\
\hline
\end{tabular}

III: - The economic assessment of the Experiment:

Data in table (6 and 7) reveal assessment of the experimental inputs and outputs as well as the ratio betweenoutputs and inputs for each treatment introducing investment ratio (IR) under the condition of Siwa Oasis, The results indicated the progressive increment in IR by increasing of Soybean Giza, 35 cultivar to combination between phosphorus fertilization, four levels as affected by four in Spraying of types of bio-fertilizers.

Found from the calculation of the economic yield of crop and oil yield, the use of the high rate of soil additive for phosphorus fertilization at the rate of 60 $\mathrm{kg}$. $\mathrm{P}_{2} \mathrm{O}_{5} /$ fed.and the bio fertilization of the Spraying with amino carpi $\mathrm{K}$ compound $200 \mathrm{~cm}^{3} / \mathrm{fed}$. is the best experimental economic transaction for the farmer under the conditions of Siwa Oasis, especially that the crop of soybeans is grown for the first time in the oasis. With the presence of other factors gave an investment rate higher than the national average, which gives a wide range of selection of transactions according to the conditions of the farmer.

Table 6. The prices of all agricultural management inputs under the condition of field experiment according to market price.

\begin{tabular}{|c|c|c|c|}
\hline Economic item & Management type & Unit & Price (L.E.) \\
\hline \multirow{9}{*}{ Input } & Bio- fertilizers & Liter/ fed. & 350 \\
\hline & Mineral fertilization $\mathrm{P}_{2} \mathrm{O}_{5}$ & Bag (50 kg./ fed.) & 75 \\
\hline & N. fertilization & Bag (50 kg./ fed.) & 150 \\
\hline & $\mathbf{K}_{2} \mathbf{O}$ & Bag (50 kg./ fed.) & 300 \\
\hline & Management operation & & 750 \\
\hline & Irrigation water & $\mathbf{M}^{3}$ & $\mathbf{0 . 7 0}$ \\
\hline & Seeds & Kg. / fed. & 120 \\
\hline & Pesticides and herbicides & Fed. & 250 \\
\hline & Agricultural rent & Fed. & 2100 \\
\hline Output & Seed yields & Kg. / fed. & 14 \\
\hline
\end{tabular}


Table7. The economic assessment of the Experiment treatments phosphorus and bio-fertilizers application of soybean on yields

\begin{tabular}{|c|c|c|c|c|c|}
\hline \multirow{2}{*}{$\begin{array}{l}\text { Bio- fertilizers } \\
\text { phosphorus fertilizers }\end{array}$} & \multirow{2}{*}{ Economic item } & \multicolumn{4}{|c|}{ Soybean } \\
\hline & & Bio.1 & Bio.2 & Bio.3 & Bio.4 \\
\hline P.1= Without & Input & 7296.1 & 6958.2 & 6865.2 & 6734.0 \\
\hline \multirow[t]{2}{*}{ fertilization } & Output & 5448.2 & 5012.0 & 5269.0 & 4883.0 \\
\hline & Investment* & 0.74 & 0.72 & 0.72 & 0.69 \\
\hline$P .2=30 \mathrm{~kg}$. & Input & 7455.2 & 7014.0 & 7113.2 & 6884.1 \\
\hline \multirow{2}{*}{ fertilization $\mathrm{P}_{2} \mathrm{O}_{5} / \mathrm{Fad}$} & Output & 5546.0 & 5158.1 & 5111.1 & 4972.1 \\
\hline & Investment* & 0.73 & 0.72 & 0.72 & 0.71 \\
\hline$P .3=45 \mathrm{~kg}$. & Input & 7669.1 & 7168.0 & 7258.1 & 6973.1 \\
\hline \multirow[t]{2}{*}{ fertilization $\mathrm{P}_{2} \mathrm{O}_{5} / \mathrm{Fad}$} & Output & 7601.2 & 6324.0 & 6124.1 & 6014.1 \\
\hline & Investment* & 0.98 & 0.88 & 1.02 & 0.86 \\
\hline \multirow{3}{*}{$\begin{array}{l}\mathrm{P} .4=60 \mathrm{~kg} \cdot \\
\text { fertilization } \mathrm{P}_{2} \mathrm{O}_{5} / \mathrm{Fad}\end{array}$} & Input & 7732.0 & 7311.0 & 7422.1 & 7002.0 \\
\hline & Output & 7856.1 & 7211.0 & 7225.2 & 6985.1 \\
\hline & Investment* & 1.01 & 0.98 & 1.12 & 0.99 \\
\hline
\end{tabular}

*Investment ratio $=$ output $/$ input $* *$ National IR = 1.32 LE output / LE input

\section{Conclusions}

The study concluded that soybean cultivation is an economic cultivation in Siwa Oasis. We recommend cultivating Giza 35 and phosphorus fertilizers at a rate of $60 \mathrm{~kg} \mathrm{p} \mathrm{p}_{2} \mathrm{O}_{5}$ fed. and bio fertilization spraying with amino karbi k., at a rate of $200 \mathrm{~cm}^{3} / 200$ L. / fed.

\section{References}

Abdel Hamid, M.T. and I. M. El-Metwally, (2008). Growth, nodulation and yield of soybean and associated weeds as affected by weed management. Planta Daninha. 26 (4): 855 - 863.

Abdel-Fattah I. El-Saharawi, Atef Z. Sabh, Sawsan M. Abou-Taleb and Ahmed E. Ghoniem., (2011).Effect of Inorganic nitrogen and Bradyrhizobiumjaponicum Inoculation on Growth and yield of Soybean. Australian J. of Basic and Appl. Sci., 5(10): 436-447.

Agwu, A. E., Ellah, J., Ekweagwu, E. and Iwuchukwu, J. C. (2009). Consumption patterns and intrahousehold roles in the production, processing and marketing of soybeans in the Northern agricultural zone of Benue State, Nigeria.African J. of Biotec., 8(4), 605-613.

Ali, S, Riaz, A. K. Ghazal, M. Arif, M. and Fida, B. (2008). Assessment of different crop nutrient management practices for yield improvement. Appl. Sci. J. of Crop Sci.,2(3), 150-157.

Amin, F. and Ezatollah G., (2014). Changes in yield and yield components of soybean (glycine max, L.) under application of Phosphate and Nitrogen Bio-fertilizers. Bull. Env. Pharmacol. Life Sci., Vol 3 Special Issue V: 98-104.

A.O.A.C. (1990).Official Methods of Analysis Official Analysis Agricular Chemists, 15th ed. Washington, D.C.U.S.A.

Carsky, R. J., Singh, B.B. and R. Oyewole (2001).Contribution of early - season cowpea to late season maize in the savanna zone of West Africa.Biol. Agric. Hort., 18, 303-315.

El-Gizawy, N. K. B. and Mehasen, S. A. S. (2009).Response of faba bean to bio, mineral phosphorus fertilizers and foliar application with zinc.World App. Sci. J., 6(10), 1359-1365.

Ghassemi-golezani, K., Taifeh-Noori, M., Oustan, Sh. And moghaddam, M. (2009). Response of soybean cultivars to salinity stress. J. \&f., Agric. \&Envi., 7:401-404.

Gordana D., Gorica C., Vojin D., Drago C., and Ljiljan, K.,(2014).Effect of microbial fertilizer on soybean yield in organic and conventional production. Turkish Journal of Agricultural and Nat. Sci. Spec.: 1(3),124- 136.

Iraj Z., Yousef S., Gholam R. H., Ali J. and Khosro M., (2012).Effects of bio fertilizers on grain yield and protein content of two soybean (Glycine max L.) cultivars. African J., of Biotec., Vol. 11(27), pp. 7028-7037.

Jackson, M.L. (1967).Soil chemical analysis.Prentice Hall, Inc. Englewood Cliffs. N. J. p. 498.

Kakar, K.M., Tariq, M., Taj, F.H. and Nawab, K. (2002).Phosphorous use efficiency of soybean as affected by phosphorous application and inoculation.Pak. J. Agron. 1(1), 49-50.

Kannayan, S. (2002). Biofertlizers for sustainable crop production, biothecnology of bio fertilizers.Narosa Publishing House, New Delhi, India, 9-49.

Katerji N., J.W. Van Hoorn, A. Hamdy, M. Mastrorilli, T. Oweis and W. Erskine (2001).Response of two varieties of lentil to soil saliniry.Agric. Water management. 47: 179-190.

Lehmann S, D. Funck, L. Szabados and D. Rentsch (2010). Proline metabolism and transport in plant development. Amino Acid. 39(4):949-962.

Manal A. A., Amal H. E.; Kh. A. S. and Abdel Mhosen, M. I.(2014). 
Influence of applied bio fertilizer on productivity, quality and nutrients content of some soybean cultivars under saline soil conditions. J.Soil Sci. and Agric. Eng., Mansoura Univ., Vol. 5 (12): 1647 - 1666.

Manal A. A., Dalia A. S. and Lamyaa A. A.(2015).Evaluation of Bio- and Mineral Phosphorus Fertilizers Accompanied with Foliar Application of Micronutrients on Quantity and Quality of Soybean (Glycin max L.)Yield. Egypt. J. Soil Sci., 22 (6):224- 237.

Sayari H. A., R. B. Gargoui, A. Bidani, L. Jaoua, A. Savouré and S. Jaoua (2005). Overexpression of 1-pyrroline-5-carboxylate synthetase increases proline production and confers salt tolerance in transgenic potato plants. Plant Sci. 169:746-752.

Sessitsch, A., J.G. Howieson, X. Perret, H. Autoun and E. Martinez- Romero.(2002). Advances
Rhizobium research. Crit. Rev. Plant, Sci. (21): 323- 378 .

Snedecor, G.W. and W.G. Cochran (1980).Statistical methods. $6^{\text {th }}$ ed. Iowa State Univ., Press, Ames, Iowa, U.S.A.

Turan, M., Afaoglu, N. and Sahin, F. (2006).Evaluation of the capacity of phosphorus solubilizing bacteria and fungi on different form of phosphorus in liquid culture.J. Sustain Agri. 28, 99-108.

Yaklich, R.W., Vinyard, B., Camp, M. and Douglass, S. (2002). Analysis of seed protein and oil from soybean northern and southern region uniform tests.Crop Sci., 42, 1504-1515.

Zaghloul, R.A., Abou-Aly, H.E., El-Meihy, R.M. and Mohamed, T. E. (2015). Improvement of growth and yield of pea plants using integrated fertilization management. Univ. J. of Agric. Res. 3 (4), 135- 143.

\section{تأثير الأسمدة الفوسفاتية والحيوية على إنتاجية وجودة فول الصويا بواحة سيوة \\ أحمد عبد المنعم عبد اللطيف عبد الوهاب \\ وحدة المحاصيل لقسم الإنتاج النباتي - مركز بحوث الطيف الصحراء}

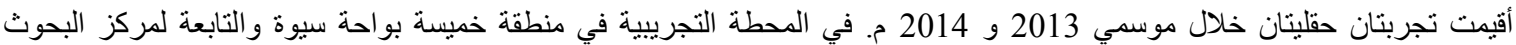

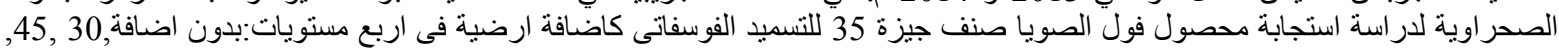

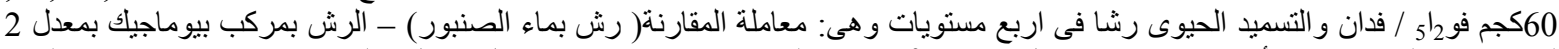

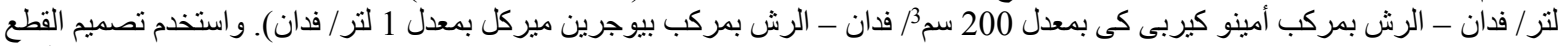
المنشقة مرة واحدة حيث وز عت معاملات الرش بارئ بالسماد الحيوى فى القطع الرئيسية , بينما التسميد الفوسفاتى فى القطع الثقية, وذللك فى أربع

$$
\text { ويمكن تلخيص النتائج فيما يلي . }
$$

1- أظهرت النتائج زيادة كبيرة فى المحصول ومكوناتة و وايضا محصول الزيت للفدان باستخدام التسميد الفوسفاتى الأرضى بمعدل 60 كجم

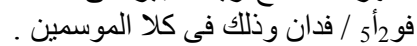

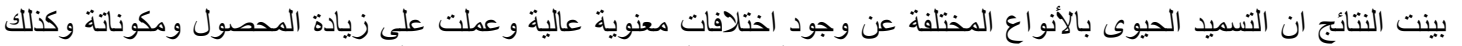

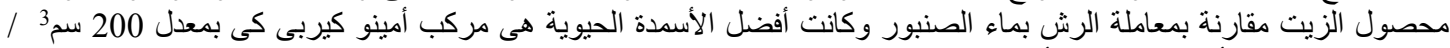

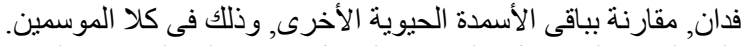

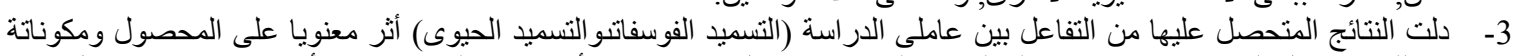

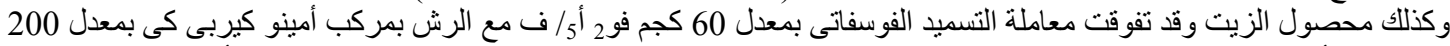

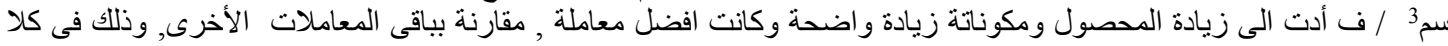
الموسمين.

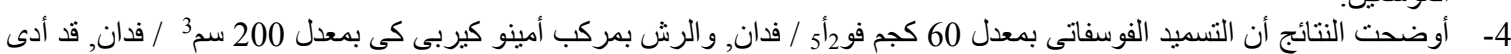



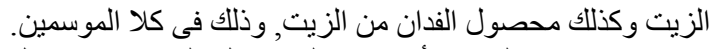

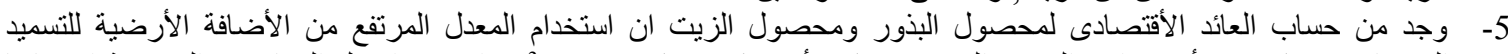

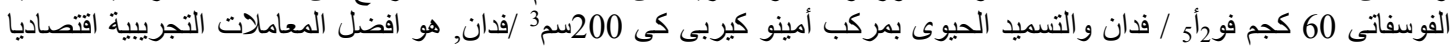

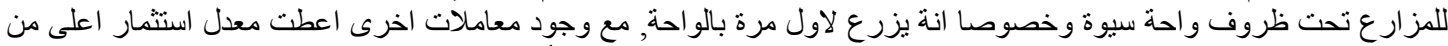



